\title{
Pulmonary versus Nonpulmonary Nontuberculous Mycobacteria, Ontario, Canada
}

\author{
Sarah K. Brode, Alex Marchand-Austin, \\ Frances B. Jamieson, Theodore K. Marras
}

In Ontario, Canada, during 1998-2010, nontuberculous mycobacteria (NTM) from pulmonary sites comprised $96 \%$ of species/patient combinations isolated; annual rates of isolation and cases increased steadily. NTM isolates from nonpulmonary sites comprised $4 \%$ of species/patient combinations; annual rates and cases were temporally stable. NTM increases were driven exclusively by pulmonary isolates and disease.

$\mathrm{N}$ ontuberculous mycobacteria (NTM) cause pulmonary and nonpulmonary disease, but most isolates and disease cases are pulmonary (1). Studies have demonstrated temporal increases in pulmonary NTM isolation and disease $(2,3)$. To determine trends in nonpulmonary NTM, we compared annual observed prevalence of pulmonary versus nonpulmonary NTM in Ontario, Canada, and compared the spectrum of NTM species isolated by body site.

\section{The Study}

We retrospectively reviewed positive NTM culture results obtained during 1998-2010 by the Public Health Ontario Laboratory, which identifies $\geq 95 \%$ of NTM isolates in Ontario (4). Until mid-2000, cultures were performed by using a Bactec 460 TB system, after which a BACTEC MGIT 960 system (Becton Dickinson, Franklin Lakes, NJ, USA) was used. DNA probes (AccuProbe; Hologic Inc., Marlborough, MA, USA) were used for speciation of Mycobacterium avium complex (MAC) and $M$. gordonae isolates; during 1998-2007, high-performance liquid chromatography was used to speciate others; thereafter, DNA probes (AccuProbe and GenoType line-probe assays [Hain Lifescience GmbH, Nehren, Germany]) were used. Because MAC was not speciated before 2008 , we used this designation throughout the study.

We counted the persons for whom $\geq 1$ positive culture for each NTM species/complex per year per body site was reported. Outcomes were pulmonary isolation

Author affiliations: West Park Healthcare Centre, Toronto, Ontario, Canada (S.K. Brode); University of Toronto, Toronto (S.K. Brode, F.B. Jamieson, T.K. Marras); University Health Network and Sinai Health System, Toronto (S.K. Brode, T.K. Marras); Public Health Ontario, Toronto (A. Marchand-Austin, F.B. Jamieson)

DOI: https://doi.org/10.3201/eid2311.170959
( $\geq 1$ positive culture from sputum, bronchoscopy samples, pleural fluid/tissue, or lung tissue); pulmonary disease ( $\geq 2$ positive sputum cultures of the same species within the calendar year, or $\geq 1$ positive culture from bronchoscopy, pleural fluid/tissue, or lung tissue (by American Thoracic Society microbiological definition, positive predictive value $70 \%-100 \%)(5-8)$; and nonpulmonary isolation ( $\geq 1$ positive culture from other sources). M. gordonae was considered a contaminant and excluded from pulmonary disease (9) but included in pulmonary isolation and nonpulmonary case counts. Outcomes were not mutually exclusive; we considered persons with NTM pulmonary disease to have pulmonary isolates and counted persons with pulmonary and nonpulmonary isolates in both groups. Careful electronic and manual selection ensured that each patient/ species/anatomic site could be represented only once per year. We calculated prevalence of annual NTM isolation and NTM pulmonary disease as the number of persons from whom NTM was isolated or who had disease in a calendar year divided by the contemporary population (Statistics Canada, http://www.statcan.gc.ca/tables-tableaux/sum-som/101/cst01/demo02a-eng.htm), expressed per 100,000 population. We used a generalized linear model with negative binomial distribution to assess annual rate changes and performed analyses with SAS version 9.4 (SAS Institute, Inc., Cary, NC, USA). All tests were 2 -tailed with a type 1 error $(\alpha)$ rate of $5 \%$. The University of Toronto Research Ethics Board approved this study.

During the study period, NTM was isolated from 26,067 patients. Pulmonary isolates predominated: mean annual unique species/patient/body site combinations were 2,631 pulmonary (96\%) and 103 nonpulmonary (4\%) (Table). Pulmonary and nonpulmonary NTM was isolated from $169(0.6 \%)$ patients. Species distributions from pulmonary versus nonpulmonary sources were similar: MAC, followed by M. xenopi, from pulmonary and nonpulmonary sites, except for $M$. marinum, which was rarely pulmonary.

Annual rates of pulmonary NTM isolation and disease prevalence were 11.4 isolates and 4.65 cases per 100,000 population in 1998 and 22.2 and 9.08 per 100,000 in 2010 (Figure 1). As reported, frequency of pulmonary isolation and disease increased steadily $(3,4)$. Annual prevalence of nonpulmonary NTM was $0.65-0.79$ isolates $/ 100,000$ and did not change appreciably over time (Figure 2 ). 
Table. Average number of patients per year who had nontuberculous mycobacteria isolated, by body site and species/complex, Ontario, Canada, 1998-2010*

\begin{tabular}{|c|c|c|c|c|c|c|c|c|}
\hline Species & Pulm & Skin/soft tissue & MS & Lymph & Blood/marrow & GI/GU & CNS & Other \\
\hline MAC & 1,328 & 8 & 1 & 2 & 17 & 18 & 0.8 & 3 \\
\hline Mycobacterium xenopi & 568 & 0.2 & 0.3 & 0 & 0.3 & 10 & 0.2 & 0.5 \\
\hline M. gordonae† & 338 & 0.2 & 0 & 0 & 0 & 6 & 0.1 & 0.3 \\
\hline M. fortuitum & 131 & 2 & 0.8 & 0 & 2 & 3 & 0.2 & 1.2 \\
\hline M. abscessus & 58 & 2 & 0.4 & 0.1 & 0.5 & 0.3 & 0 & 0.8 \\
\hline M. chelonae & 40 & 3 & 1 & 0.2 & 0.5 & 0.7 & 0 & 1.0 \\
\hline M. simiae complex & 42 & 0.6 & 0.3 & 0.1 & 0.5 & 0.7 & 0 & 0.5 \\
\hline M. kansasii & 34 & 0.3 & 0.2 & 0 & 0.2 & 0.6 & 0 & 0.1 \\
\hline M. marinum & 0 & 6 & 1.5 & 0 & 0 & 0 & 0 & 0.6 \\
\hline Other & 92 & 0.8 & 0.5 & 0.1 & 1 & 2 & 0.1 & 0.3 \\
\hline All & 2,631 & 22 & 6 & 3 & 22 & 41 & 1 & 8 \\
\hline
\end{tabular}

*Contemporary Ontario population 11.3-13.2 million. CNS, central nervous system; GI, gastrointestinal system; GU, genitourinary system; lymph, lymphatic system; MAC, Mycobacterium avium complex; MS, musculoskeletal system; pulm, pulmonary system.

†M. gordonae was excluded from the number of pulmonary disease cases but included in pulmonary and nonpulmonary isolation cases.

†Other species most commonly identified ( $\%$ of grand total over entire study period, average no. patients per year), for pulmonary isolates were $M$.

mucogenicum $(0.93 \%, 24.5)$, M. terrae complex $(0.43 \%, 11.4)$, M. scrofulaceum $(19 \%, 5), M$. peregrinum (0.17\%, 4.4), M. neoaurum (0.14\%, 3.8), $M$.

shimoidei $(0.13 \%, 3.3)$, M. szulgai $(0.12 \%, 3.2)$, M. celatum $(0.09 \%, 2.4)$, M. malmoense $(0.08 \%, 2.2), M$. elephantis $(0.08 \%, 2.1)$, and $M$. margeritense/M. smegmatis (0.06\% each, 1.5 each) and for nonpulmonary isolates included $M$. mucogenicum (1.3\%, 1.38), M. smegmatis (1.0\%, 1.08), M. terrae complex $(0.5 \%, 0.54), M$. genavense $(0.4 \%, 0.38), M$. senegalense $(0.2 \%, 0.23), M$. malmoense/M. scrofulaceum $/ M$. szulgai $(0.15 \%$ each, 0.15 each), $M$. margeritense/M. shimoidei /M. elephantis ( $0.07 \%$ each, 0.08 each).

\section{Conclusions}

Contrasting with documented increased pulmonary NTM isolation and disease in Ontario, rates of nonpulmonary NTM isolation are stable or decreasing, regardless of species or body site. The difference in trends indicates that pulmonary and nonpulmonary NTM represent different diseases with different risk factors. NTM pulmonary disease generally occurs in persons with preexisting structural lung damage (8) or abnormal mucociliary function (10) and is strongly associated with increasing age (5). Nonpulmonary NTM disease occurs in persons with generalized immunosuppression or after NTM entry into breached tissue (9). We considered the possibility that after the introduction of antiretroviral medication for HIV in the 1990s, a reduction in disseminated NTM may have masked an increase in nonpulmonary NTM infection in non-HIV-infected patients. However, the absence of decreased isolation from blood or bone marrow in the first several years and the steady number of cases (Figure 2) do not support this possibility. Although we lacked data regarding immune status, the small proportion of patients from whom pulmonary and nonpulmonary NTM were isolated $(0.6 \%)$ does not suggest a large proportion of severely immunosuppressed patients.

There are several possible explanations for discordant temporal trends between pulmonary and nonpulmonary NTM. A selective increase in respiratory exposure would

Figure 1. Prevalence of pulmonary and nonpulmonary nontuberculous mycobacteria (NTM) isolation and pulmonary NTM disease in Ontario, Canada, 1998-2010. Annual increase and modeled annual change were $6.3 \%(3,4)$ and 1.04 $(95 \%$ Cl 0.696-1.38)/100,000 population $(p<0.001)$ for pulmonary isolation and $8.0 \%(3)$ and 0.402 (95\% Cl 0.307-0.497)/100,000 population $(p<0.001)$ for pulmonary disease. Significant increases occurred in Mycobacterium avium complex (annual change 0.291 [95\% Cl 0.236-0.346]/100,000 population; $p<0.001) ; M$. xenopi (annual change $0.059[95 \% \mathrm{Cl}$ $0.015-0.103] / 100,000$ population; $p=0.002$ ); and $M$. abscessus (annual change $0.019[95 \% \mathrm{Cl}$ 0.015-0.024]/100,000 population;

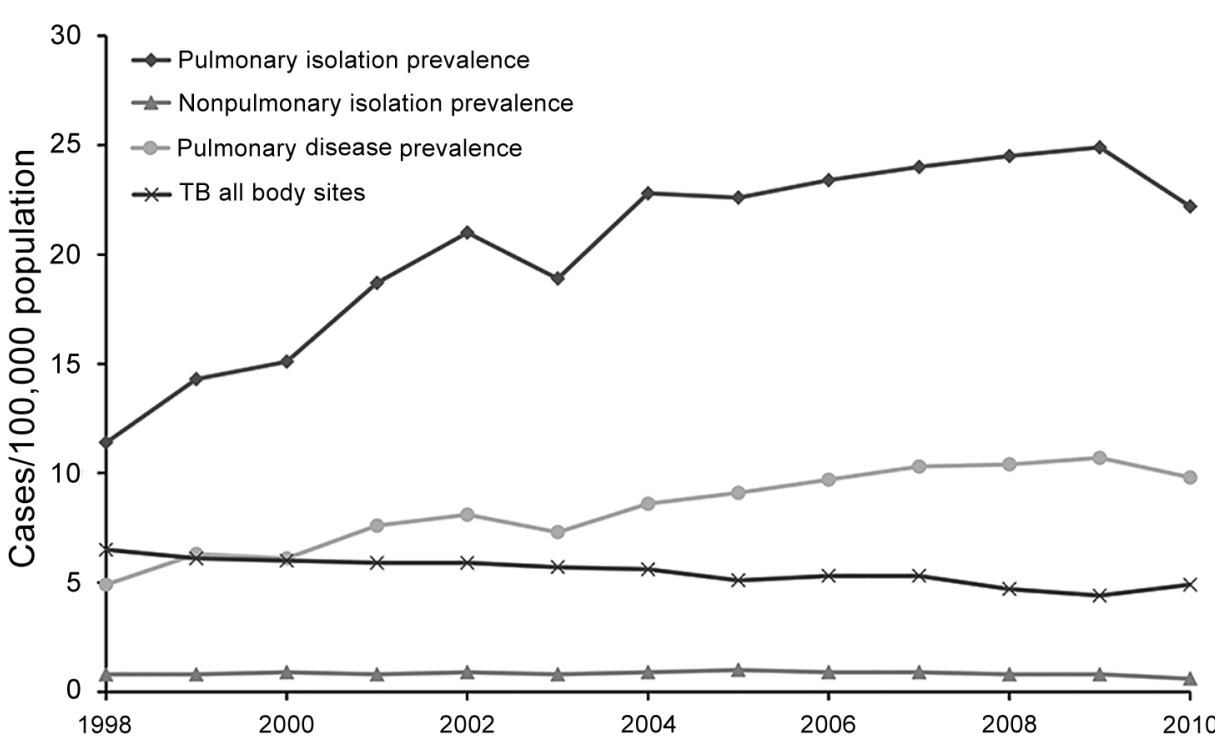
$\mathrm{p}<0.001)$. TB (all body sites) isolation decreased by an average of $2.2 \%$ annually ( 6.5 to $4.9 / 100,000$ population) during the study period. TB, tuberculosis. 
Figure 2. Isolation of nonpulmonary nontuberculous mycobacteria by body site, Ontario, Canada, 1998-2010. There was no significant temporal change by anatomic site except for a decrease in skin/soft tissue infections (modeled annual change $-0.011[95 \% \mathrm{Cl}-0.020$ to -0.003$] / 100,000$ population; $p=0.001)$. Mycobacterium marinum significantly decreased over time (modeled annual change $-0.003[95 \% \mathrm{Cl}-0.007$ to $0.001] / 100,000$ population; $p=$ 0.0480 ); isolation of other species from nonpulmonary sites was unchanged. Overall nonpulmonary isolation modeled annual change was $-0.004(95 \% \mathrm{Cl}-0.019$ to $0.010) / 100,000$ population $(p=$ $0.410)$. CNS, central nervous system; GI, gastrointestinal system; GU, genitourinary system; lymph, lymphatic system; MS, musculoskeletal system.

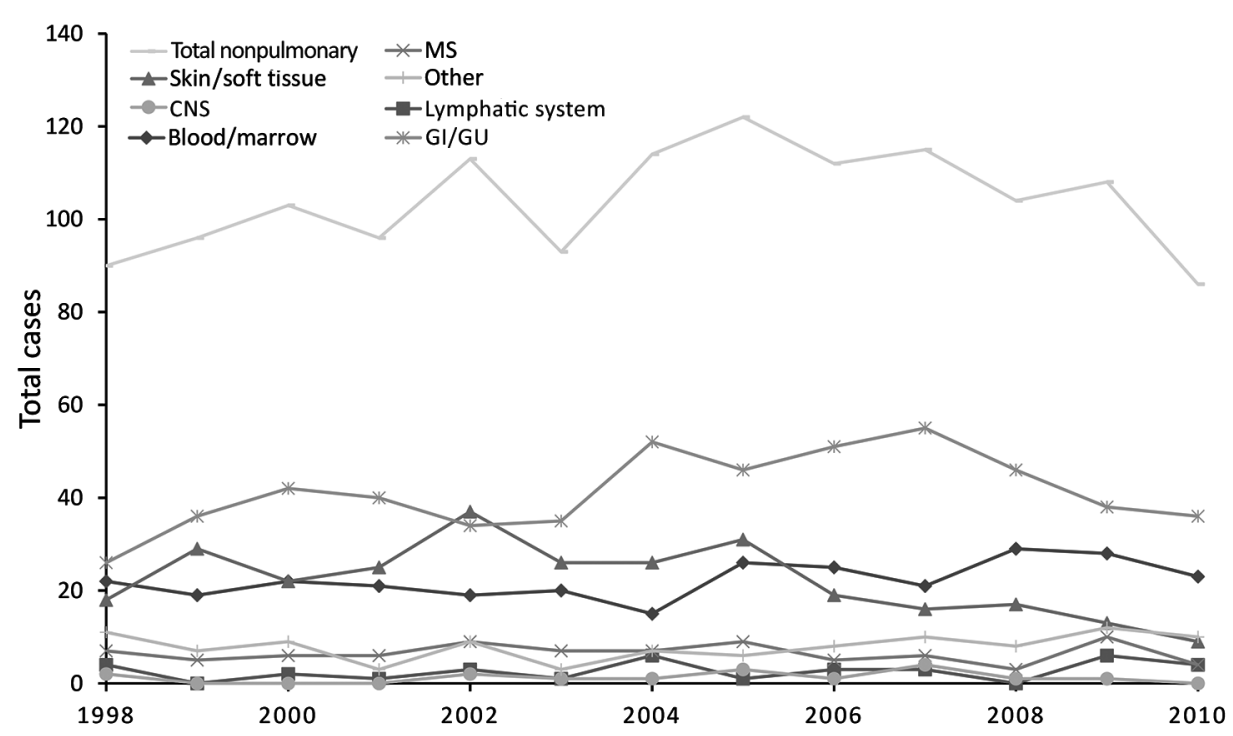

preferentially drive pulmonary NTM. However, although exposure to respirable waterborne NTM is undoubtedly widespread, data identifying recent increases are lacking. Other possibilities include changes in risk factors for NTM pulmonary disease (e.g., aging, chronic obstructive pulmonary disease, and iatrogenic causes [medications]) and improved diagnostic modalities (e.g., increased use of computed tomography in the United States [11]). Reduced turberculosis incidence, leading to reduced cross-immunity to NTM, has been proposed as an explanation for the reciprocal trends in tuberculosis and NTM infections observed in many areas (2); however, it is not known why waning immunity would result in increased pulmonary NTM only.

A previous population-based study compared the epidemiology of pulmonary and nonpulmonary NTM by examining isolates referred to the Netherlands national reference laboratory during 2000-2007 (12). The study indicated large increased numbers of NTM isolates, mostly $M$. avi$u m$. The average annual percentage increase was similar for pulmonary $(31.3 \%)$ and extrapulmonary $(33.0 \%)$ M. avium, differing markedly from our results. The differences could reflect referral bias (the Netherlands national reference laboratory received $85 \%$ of NTM isolates; the Public Health Ontario Laboratory received $>95 \%$ ), improvements in laboratory methods in the Netherlands potentially increasing detection of both pulmonary and nonpulmonary NTM, or differences in NTM epidemiology by region $(13,14)$.

In Olmsted County, Minnesota, USA, incidence of cutaneous NTM infections apparently tripled from 19801999 to 2000-2009, driven partly by increased M. abscessus/chelonae, often from surgical/cosmetic procedures
(15). Contrasting with our observation of reduced skin/ soft tissue infections, their observations could result from differences in methods, geography, or number of surgical/ cosmetic procedures.

A study limitation is lack of clinical data to confirm NTM pulmonary disease. Although our definition of NTM pulmonary disease has acceptable positive predictive value (5-8), it misclassifies some patients as having disease. By contrast, we underestimated NTM pulmonary disease because we counted only persons who met the definition each calendar year; there were probably persons with prevalent disease that was undiagnosed or diagnosed in a previous year but lacked ongoing sputum collection or for whom NTM isolation was staggered over 2 calendar years. The net effect of over/underestimating NTM pulmonary disease is unclear. We also classified all nonpulmonary isolates as representing disease, overestimating nonpulmonary disease, especially because feces and urine comprise most gastrointestinal/genitourinary sources. Excluding gastrointestinal/genitourinary isolates would increase the proportion of pulmonary isolations from $96.0 \%$ to $97.7 \%$. Although gastrointestinal/genitourinary isolates comprise the largest nonpulmonary group, the trend for this group is similar to that for others (Figure 2), so its inclusion does not affect our conclusions. The lack of data regarding the number of samples submitted prevents assessment of the effect of sampling on NTM isolation. However, we previously identified an increased number of pulmonary samples submitted during 1997-2002, leveling off during 2002-2007 (4). Given the steady increase in pulmonary isolates and disease throughout the study 
period, increased sampling does not explain our findings. Our lack of clinical data prevents comparison of features of pulmonary versus nonpulmonary NTM, and our lack of detailed epidemiologic data prevents assessment for regional or temporal nonpulmonary outbreaks from common point sources. The increase of NTM in Ontario reflects only pulmonary NTM.

Dr. Brode is an attending staff physician in the Division of Respirology, Department of Medicine, at the University Health Network, Sinai Health System, and West Park Healthcare Centre, Toronto, and assistant professor of medicine,

University of Toronto, Canada. Her research interests are NTM and tuberculosis.

\section{References}

1. Marras TK, Chedore P, Ying AM, Jamieson F. Isolation prevalence of pulmonary non-tuberculous mycobacteria in Ontario, 1997-2003. Thorax. 2007;62:661-6. http://dx.doi.org/10.1136/ thx.2006.070797

2. Brode SK, Daley CL, Marras TK. The epidemiologic relationship between tuberculosis and non-tuberculous mycobacterial disease: a systematic review. Int J Tuberc Lung Dis. 2014;18:1370-7. http://dx.doi.org/10.5588/ijtld.14.0120

3. Marras TK, Mendelson D, Marchand-Austin A, May K, Jamieson FB. Pulmonary nontuberculous mycobacterial disease, Ontario, Canada, 1998-2010. Emerg Infect Dis. 2013;19:1889-91. http://dx.doi.org/10.3201/eid1911.130737

4. Al Houqani M, Jamieson F, Chedore P, Mehta M, May K, Marras TK. Isolation prevalence of pulmonary nontuberculous mycobacteria in Ontario in 2007. Can Respir J. 2011;18:19-24. http://dx.doi.org/10.1155/2011/865831

5. Prevots DR, Shaw PA, Strickland D, Jackson LA, Raebel MA, Blosky MA, et al. Nontuberculous mycobacterial lung disease prevalence at four integrated health care delivery systems. Am J Respir Crit Care Med. 2010;182:970-6. http://dx.doi.org/10.1164/ rccm.201002-0310OC

6. Andréjak C, Thomsen VO, Johansen IS, Riis A, Benfield TL, Duhaut $\mathrm{P}$, et al. Nontuberculous pulmonary mycobacteriosis in Denmark: incidence and prognostic factors. Am J Respir Crit Care Med. 2010;181:514-21. http://dx.doi.org/10.1164/ rccm.200905-07780C
7. Winthrop KL, McNelley E, Kendall B, Marshall-Olson A, Morris C, Cassidy M, et al. Pulmonary nontuberculous mycobacterial disease prevalence and clinical features: an emerging public health disease. Am J Respir Crit Care Med. 2010;182:97782. http://dx.doi.org/10.1164/rccm.201003-0503OC

8. Marras TK, Mehta M, Chedore P, May K, Al Houqani M, Jamieson F. Nontuberculous mycobacterial lung infections in Ontario, Canada: clinical and microbiological characteristics. Lung. 2010;188:289-99. http://dx.doi.org/10.1007/s00408-010-9241-8

9. Griffith DEAT, Aksamit T, Brown-Elliott BA, Catanzaro A, Daley C, Gordin F, et al.; ATS Mycobacterial Diseases Subcommittee; American Thoracic Society; Infectious Diseases Society of America. An official ATS/IDSA statement: diagnosis, treatment, and prevention of nontuberculous mycobacterial diseases. Am J Respir Crit Care Med. 2007;175:367-416. http://dx.doi.org/10.1164/rccm.200604-571ST

10. Fowler CJ, Olivier KN, Leung JM, Smith CC, Huth AG, Root H, et al. Abnormal nasal nitric oxide production, ciliary beat frequency, and Toll-like receptor response in pulmonary nontuberculous mycobacterial disease epithelium. Am J Respir Crit Care Med. 2013;187:1374-81. http://dx.doi.org/10.1164/rccm.201212-2197OC

11. Gould MK, Tang T, Liu IL, Lee J, Zheng C, Danforth KN, et al. Recent trends in the identification of incidental pulmonary nodules. Am J Respir Crit Care Med. 2015;192:1208-14. http://dx.doi.org/10.1164/rccm.201505-0990OC

12. van Ingen J, Hoefsloot W, Dekhuijzen PN, Boeree MJ, van Soolingen D. The changing pattern of clinical Mycobacterium avium isolation in the Netherlands. Int J Tuberc Lung Dis. 2010;14:1176-80.

13. Chou MP, Clements AC, Thomson RM. A spatial epidemiological analysis of nontuberculous mycobacterial infections in Queensland, Australia. BMC Infect Dis. 2014;14:279. http://dx.doi.org/10.1186/1471-2334-14-279

14. Adjemian J, Olivier KN, Seitz AE, Falkinham JO III, Holland SM, Prevots DR. Spatial clusters of nontuberculous mycobacterial lung disease in the United States. Am J Respir Crit Care Med. 2012;186:553-8. http://dx.doi.org/10.1164/rccm.201205-0913OC

15. Wentworth AB, Drage LA, Wengenack NL, Wilson JW, Lohse CM. Increased incidence of cutaneous nontuberculous mycobacterial infection, 1980 to 2009: a population-based study. Mayo Clin Proc. 2013;88:38-45. http://dx.doi.org/10.1016/j.mayocp.2012.06.029

Address for correspondence: Sarah K. Brode, Toronto Western Hospital, 7th Floor, East Wing, 399 Bathurst St, Toronto, ON M5T 2S8, Canada; email: sarah.brode@uhn.ca

\section{$\underset{\text { MDUCATION }}{\text { Medscape }}$ Sign up to recieve email announcements CME when a new article is available.}

\section{Get an online subscription at wwwnc.cdc.gov/eid/subscribe.htm}

